\title{
Evolución y perspectivas del sistema de abastecimiento de la ciudad de Santiago de Cali frente al aseguramiento de la calidad del agua potable
}

SANITARY AND ENVIRONMENTAL ENGINEERING

\section{Evolution and perspectives of the water supply system in the city of Santiago de Cali front of the water quality insurance}

\author{
Andrea Pérez-Vidal*, Luis G. Delgado-Cabrera**, Patricia Torres-Lozada* $§$ \\ * Grupo de Investigación Estudio y Control de la Contaminación Ambiental, Universidad del Valle \\ ** Departamento de Producción de Agua Potable, EMCALI EICE ESP, Cali, Colombia
}

§patricia.torres@correounivalle.edu.co,perezvid@univalle.edu.co

(Recibido: Agosto 11 de 2011 - Aceptado: Noviembre 19 de 2012)

\begin{abstract}
Resumen
La ciudad de Santiago de Cali, fundada en el año 1536 inició su crecimiento y desarrollo en torno al río Cali; en 1778 se fundó el primer acueducto que surtía de agua a los habitantes de la época mediante pilas públicas y a final de la década de los 50's el río Cauca se convirtió en la principal solución a la constante crisis de desabastecimiento de la ciudad. Este artículo de reflexión pretende analizar la evolución que ha tenido el Sistema de Abastecimiento de Agua Potable (SAAP) de la ciudad, las mejoras y ampliaciones acordes a los requerimientos de crecimiento y desarrollo, el estado actual y las medidas de control implementadas para garantizar permanentemente el suministro de agua segura a los usuarios. Actualmente, la empresa prestadora del servicio de agua ha implementado un Sistema de Gestión de la Calidad y viene desarrollando un Plan de Seguridad del Agua (PSA) como herramientas de gestión del riesgo sanitario y aseguramiento de la calidad del agua potable, dando respuesta a las nuevas tendencias mundiales y considerando un enfoque integral de todos los componentes del SAAP (fuente, procesos de tratamiento, distribución y usuario final).
\end{abstract}

Palabras Clave: Calidad de agua potable, Plan de Seguridad del Agua, Riesgo sanitario, Sistema de abastecimiento de agua potable.

\begin{abstract}
The city of Santiago de Cali was founded in 1536 and began its development and expansion around the Cali river; in 1778 was founded the first aqueduct that supplied water to people of the time by public piles and at the end of the decade of 50's, the Cauca river became the main solution to constant shortage crisis in the city. This reflection paper aims to analysis the evolution of that has taken the Water Supply System (WSS) of the city, the improvements and additions in line with the requirements of growth and development, the current status and control measures implemented to permanently ensure safe water supply to consumers. Currently, the water service company has implemented a Management System and Quality and is developing a Water Safety Plan (WSP) as tools for health risk management and quality assurance of drinking water, responding to the emerging global trends and considering an integral approach of all WSS-steps (water source, treatment processes, distribution system and consumer).
\end{abstract}

Keywords: Drinking water quality, Sanitary Risk, Water Safety Plan, Water Supply System. 


\section{Introducción}

El agua es un recurso esencial para la vida y preservación de ecosistemas y biodiversidad; su su potabilización adquiere mayor valor por estar orientado a la protección de la salud de la población. El suministro de agua segura gira en torno a las metas de los Objetivos de Desarrollo del Milenio (ODM) que contemplan a 2015 reducir a la mitad el porcentaje de personas que carecen de acceso sostenible a agua potable segura y saneamiento básico (Objetivo 7, Meta 10). Sin embargo, más de 1,200 millones de personas en el mundo no tienen acceso a agua segura y las enfermedades relacionadas por la falta de agua o calidad inadecuada afectan a más de 2,300 millones (Rojas, 2006). En Colombia mueren anualmente 1,300 niños por enfermedades diarreicas causadas por consumir agua no potable (MAVDT, 2008a) y sólo el $65 \%$ de la población cuenta con eficiente cobertura de acueducto y alcantarillado (MAVDT, 2008b).

Las mejoras de calidad del agua por sí solas reducen en un tercio o más la morbilidad debida a enfermedades diarreicas (OMS, 2007), recomendándose la adopción de los Planes de Seguridad del Agua (PSA) (OMS, 2004) como una herramienta preventiva que integra conceptos de evaluación y gestión del riesgo en Sistemas de Abastecimiento de Agua Potable (SAAP) y promueven la protección de la salud humana mediante el aseguramiento de la calidad del agua desde la cuenca de abastecimiento hasta el usuario final (WHO, 2008); su implementación en los SAAP permite tomar medidas oportunas frente a la presencia de peligros microbiológicos o químicos antes de que el agua contaminada llegue al consumidor, protegiendo de este modo la salud de la población (Godfrey \& Howard, 2005).

El SAAP de la ciudad de Santiago de Cali, a cargo de las Empresas Municipales de Cali - EMCALI EICE ESP, ha experimentado cambios a través de la historia encaminados al aseguramiento de la calidad y cantidad de agua suministrada, respondiendo a los permanentes cambios asociados al desarrollo de la ciudad y variaciones en las fuentes de abastecimiento.
Actualmente la ciudad es abastecida por cuatro ríos mediante cinco plantas de tratamiento $\mathrm{y}$ cuatro sistemas de distribución de agua potable.

En este artículo de reflexión se muestra la evolución del SAAP de la ciudad de Cali, analizando las mejoras y ampliaciones al sistema para hacer frente al rápido crecimiento y desarrollo de la ciudad y a los requerimientos de suministro de agua potable para asegurar la salud de la población; adicionalmente, se presentan las perspectivas y tendencias de la empresa prestadora del servicio de agua potable con relación al aseguramiento de la calidad del agua desde la cuenca de abastecimiento hasta el usuario final.

\section{Evolución del sistema deabastecimiento de agua potable de la ciudad de Cali}

Hacia 1574, la ciudad (1,500 habitantes) se abastecía del río Cali por una pequeña acequia que corría por la Calle La Merced y mediante derivaciones en guadua, se surtían los predios más importantes (Pérez, 2006; EMCALI s.f). El primer acueducto - Acueducto Colonial - se inauguró en 1796 y terminó su construcción en 1824 y abastecía a la ciudad con pilas públicas, obras adelantadas con materiales como calicanto y ladrillo bajo la dirección del Ing. español Juan Antonio Monzón; la bocatoma se ubicó en el río Cali, $1 \mathrm{Km}$ arriba del puente de Santa Rosa (parte alta de la ciudad - Colina de San Antonio) y conducía el agua hasta la antigua fábrica de Aguardiente (Cra $4^{\mathrm{a}}$ - Calles 13 y 14) y las pilas San Pedro, Jaime, Crespo y Lores (EMCALI s.f); Aladino, 1992; Vásquez et al, 1995).

A principios del Siglo XX (1900 -1930), la ciudad aún se abastecía del Acueducto Colonial (Directorio Telefónico 2005-2006). En 1903 se construyeron nuevos tramos y pilas (la Chanca, Matadero, Belalcázar, Buenaventura y González) y un tramo del canal principal que partía de la bocatoma se derrumbó, mostrando la vulnerabilidad del sistema y la necesidad de construir un acueducto metálico (Vásquez, 2008). La Figura 1 muestra el esquema del Acueducto Colonial de la época. 


\section{ACUEDUCTO COLONIAL HASTA 1903}

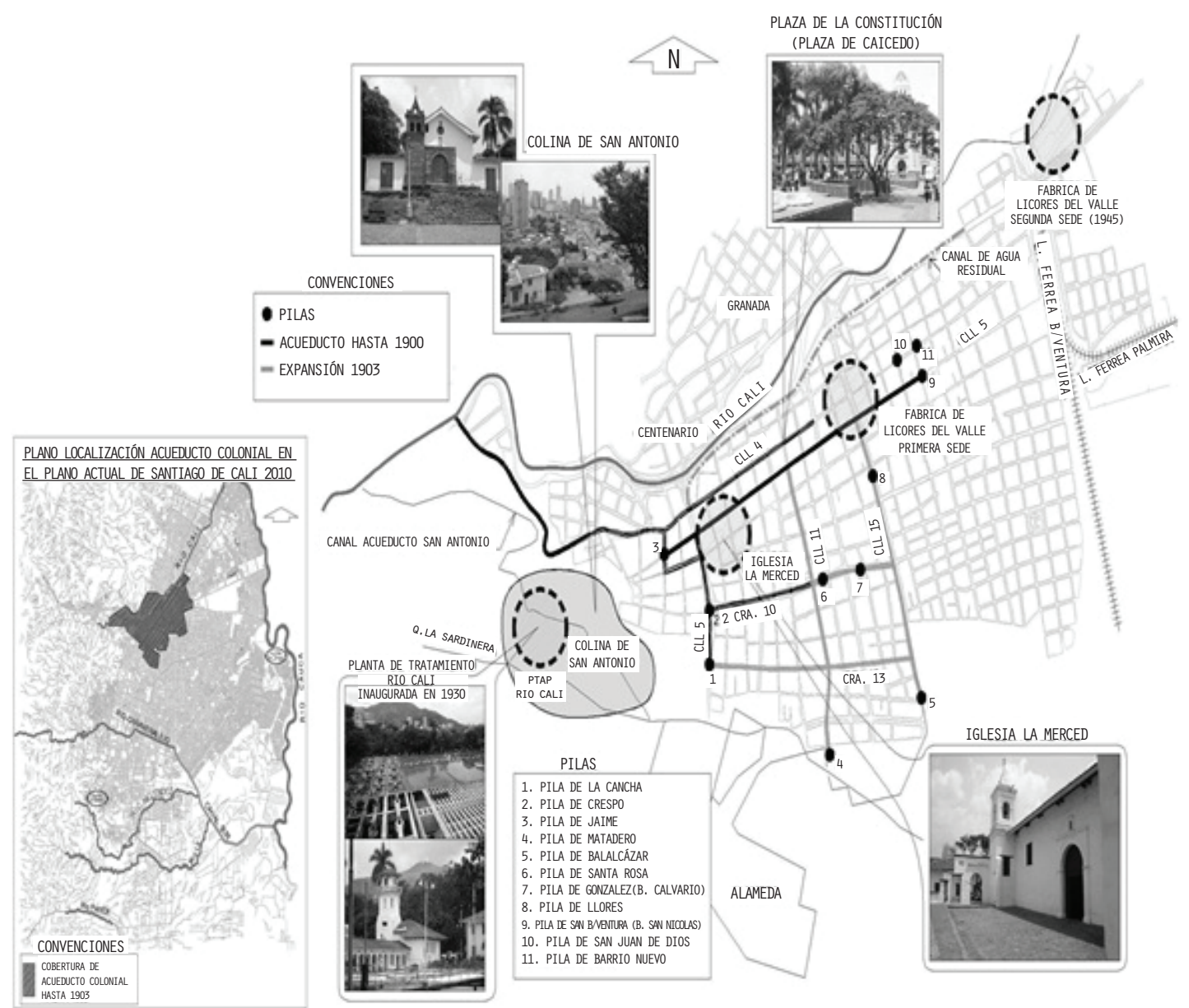

Figura 1. Acueducto Colonial de la ciudad de Santiago de Cali hasta el año 1903. Fuente: (Adaptado Vásquez et al, 1995; Patiño 2008)

En enero de 1915 se inauguró el Ferrocarril del Pacífico y se extendió la infraestructura fluvial (barco a vapor por el río Cauca) que favoreció el desarrollo económico de la región y facilitó el transporte de mercancía y pasajeros (Vásquez, 2001; Centro virtual Isaacs, 2004; Camacho, 2006). El desarrollo naviero en el río Cauca indujo la explotación maderera y tala de bosques, liberando extensas tierras cultivables en que se sembró principalmente caña de azúcar, actividades que fueron convirtiendo las orillas y las planicies inundables del río en zonas agrícolas y ganaderas (Sandoval \& Ramírez, 2007). Vásquez (2001) resalta que la variabilidad del caudal del río, asociado a los periodos climáticos de verano e invierno, se registraron como una limitación para la navegación a vapor.
Debido a que el agua era conducida por acequias expuestas a toda clase de contaminantes, las enfermedades intestinales y principalmente la fiebre tifoidea fueron endémicas en la población (Vásquez et al, 1995; Vásquez, 2008) y en 1911 el Concejo Municipal (Acuerdo 020) aprobó la construcción del acueducto metálico a presión, estudio a cargo de Ferrocarril del Pacífico (EMCALI s.f; Aladino, 1992).

En 1916, se creó la Junta Constructora del Acueducto Municipal (JCAM) (Vásquez, 2008), la primera etapa duró 12 años e incluyó la bocatoma sobre el río Cali aguas arribas de planta eléctrica $\mathrm{N}^{\circ} 1$, un canal de conducción abierto en forma trapezoidal y dos estanques en la colina de San Antonio que garantizaban la presión suficiente (EMCALI s.f; Vásquez et 
al, 1995). En 1919 se instaló la primera red de acueducto en tubería de hierro y se instalaron hidrantes cada dos cuadras que permitían apagar los frecuentes incendios de las casas con techos de paja, principalmente en época de verano (Vásquez et al, 1995).

Aunque el cambio del acueducto colonial al metálico mejoró la calidad del agua, no fue suficiente para lograr el impacto esperado en la salud de la población pues el canal de conducción abierto era susceptible de contaminación, presentándose frecuentes epidemias de enfermedades de origen hídrico como fiebre tifoidea, paratifoidea, disentería bacilar y amebiana, gastroenteritis y cólera, lo que mostraba la urgente necesidad de construir una planta de tratamiento (Vásquez, 2008; Vásquez et al, 1995).

En 1926, la JCAM agrupó varias empresas del municipio para darle un fuerte impulso a la reforma y ampliación del acueducto (diseño y construcción a cargo del Ing. norteamericano George Bunker) (Mercado, 2006); mediante Acuerdo 9 de Marzo de 1930, se integraron en una sola entidad pública la Empresa del Acueducto Municipal, la Plaza de mercado y el matadero, nombrando como primer Gerente de la entidad al Dr. Joaquín Borrero Sinisterra (Mercado, 2006; Directorio 2005-2006).

La selección de la tecnología de filtración lenta (Europa) o convencional(USA), causó una fuerte discusión en el Concejo, pero las inspecciones del equipo internacional (G. Bunker, Lewis B. Moore y Eugene Chartier) a la cuenca que hacían prever su grave deterioro y el acelerado crecimiento de la población, motivaron la selección de la tecnología convencional y la recomendación de planificar y establecer fuertes acciones para la conservación de la cuenca del río Cali tales como prohibir la tala de árboles y hacer jornadas de reforestación (Vásquez et al, 1995).

En 1927 se inició la construcción de la Planta de Tratamiento de San Antonio (Patiño, 2008), que fue inagurada el 31 de Diciembre de 1930 $\left(33,000 \mathrm{~m}^{3} / \mathrm{día}-0.38 \mathrm{~m}^{3} / \mathrm{s}\right)$. El Acuerdo $13 \mathrm{de}$ 1931 delegó la administración del acueducto a una Junta Administradora del Agua constituida por tres miembros que prácticamente dio origen a las Empresas Municipales de Cali (Mercado, 2006; Camacho, 2006). Los efectos positivos en la salud de la población fueron evidentes; en 1930 los casos de muerte por disentería (258) y fiebre tifoidea (122) bajaron en 1938 (con una población $60 \%$ mayor) a 9 y 17 respectivamente (Vásquez, 2008).

El término de la crisis económica mundial (1929 - 1932) y la disponibilidad de agua potable, favorecieron la industrialización de la ciudad y su rápido crecimiento poblacional (Vásquez, 2001), lo que aunado a la falta de conciencia de uso racional del agua, agotó rápidamente la capacidad de la planta de tratamiento, siendo necesario realizar a partir de 1940 ampliaciones sucesivas para elevar su capacidad entre 42,000 y $69,911 \mathrm{~m}^{3} /$ día (Magazine Despertar Vallecaucano, 1976). Hacia 1941 se preveía la crisis de desabastecimiento asociada a las intensas sequías y al elevado deterioro de la cuenca del río Cali ya anunciado por el Ing. Bunker, quien sugirió buscar nuevas fuentes (ríos Aguacatal, Meléndez y Anchicayá) y reducir consumos de agua (Vásquez et al, 1995); en 1943 se implementaron medidores de agua con cobertura de $97 \%$, aunque la medida fue insuficiente y la crisis continuó (Vásquez et al, 1995).

El río Cauca quedaba muy retirado del casco urbano, se encontraba a muy baja altura que implicaba bombeo y su calidad era inferior a la del río Cali, por lo que no fue considerado hasta ese momento; siguiendo las recomendaciones de Bunker, las empresas municipales contrataron los estudios mostrados en la Tabla 1. Los elevados costos de las dos primeras opciones inclinaron la solución hacia la tercera y en 1952 perforaron pozos de mediana profundidad en los barrios Aguablanca, Villanueva y Nápoles que abastecieron de forma inmediata el área desabastecida; sin embargo, por el continuo crecimiento de la ciudad y el agotamiento de la capacidad de los pozos, el problema de desabastecimiento continuó.

En 1953, el Ing. Bunker recomendó por primera vez utilizar el río Cauca para mejorar la oferta de 
Tabla 1. Opciones evaluadas en la década de los 40's para resolver la crisis de desabastecimiento de la ciudad.

\begin{tabular}{ccl}
\hline $\begin{array}{c}\text { Estudio } \\
\mathrm{N}^{\mathrm{o}}\end{array}$ & \multicolumn{1}{c}{ Nombre } & \multicolumn{1}{c}{ Objetivo } \\
\hline 1 & Sistema de presas de embalses y regulación del \\
caudal del río Cali & $\begin{array}{l}\text { Buscaba aumentar la capacidad de suministro mediante embalses y la } \\
\text { ampliación de la planta de San Antonio. }\end{array}$ \\
2 & Aprovisionamiento de las aguas de la vertiente \\
del Pacífico & $\begin{array}{l}\text { Contemplaba un acueducto desde la cuenca del río Cava con la construcción } \\
\text { de 4.6 Km de túnel, 11 Km de canal principal y 13 Km de canal secundario. } \\
\text { La construcción de túneles y canales, aún pudiéndose generar energía a } \\
\text { partir de las caídas de agua, hicieron inviable económicamente el proyecto. } \\
\text { Proponía la extracción de agua subterránea por medio de pozos utilizando } \\
\text { bombeo para captar 20,000 m3/día y empleando filtros para purificar el } \\
\text { agua. }\end{array}$ \\
\hline
\end{tabular}

Fuente: adaptado de Vásquez et al (1995).

agua de la ciudad. Los estudios y construcción de la Planta Río Cauca fueron contratados con Establisements Emile Degrémont (inicio construcción en 1955: 89,014 m³/día - $1.03 \mathrm{~m}^{3} / \mathrm{s}$ ), junto con la ampliación de la Planta San Antonio $\left(86,400 \mathrm{~m}^{3} / \mathrm{d}-1.0 \mathrm{~m}^{3} / \mathrm{s}\right)$; ambos proyectos entraron en operación en 1958, saliendo de funcionamiento los pozos (EMCALI, s.f; Vásquez et al, 1995; Patiño, 2008); además, se construyó el tanque de almacenamiento de agua tratada La Normal $\left(15,000 \mathrm{~m}^{3}\right)$ y su respectiva conducción para la transferencia de agua a la red baja en épocas de abundancia en el río Cali, la red Principal Sur (1959) y redes domiciliarias para los barrios del sur y suroriente (Vásquez et al, 1995; Vásquez, 2008).

La tecnología seleccionada se fundamentó en la variabilidad de la calidad del agua del río Cauca que en invierno inundaba gran parte del Valle Geográfico (limitaciones por turbiedad) y en verano presentaba niveles bajos (caudales mínimos que impedían diluir la creciente contaminación de la época), siendo seleccionados clarificadores de contacto con agitación mecánica, que además de haber sido probados con éxito en varios países eran eficientes para remover material particulado; su diseño y construcción se basó en un modelo de planta que operaba en Marruecos (Patiño, 2008). Desde esta planta se tendieron dos grandes redes de conducción, una para abastecer el sur y el oriente de la ciudad y la otra para abastecer el norte; desde entonces el suministro de agua en la ciudad quedó dividido en la Red Alta (abastecida por la Planta San Antonio) y la Red Baja (abastecida por la Planta Río Cauca) (Camacho, 2006).
Los desastres ocasionados por las inundaciones del río Cauca y el desbordamiento de sus afluentes, que fomentaron el origen de la Corporación Autónoma Regional del Valle del Cauca (CVC) en 1954 (CVC, 2011), fueron en parte mitigados con la construcción del Jarillón margen izquierdo del río Cauca en 1960, con el cual se buscó proteger la zona baja de la ciudad frente a inundaciones y aprovechar la tierra en actividades agrícolas; posteriormente en esta zona se presentaron asentamientos humanos, constituyéndose el Distrito de Aguablanca; con la construcción del Jarillón se empleó gran cantidad de tierra, parte de la cual se extrajo de la zona donde posteriormente se ubicaría el botadero a cielo abierto de Navarro en 1969.

Los constantes desbordamientos de los ríos Cañaveralejo, Lilí y Meléndez, que ocasionaban grandes inundaciones en las zonas del oriente y sur-oriente de la ciudad, también propiciaron que la CVC entre 1958 y 1962, construyera un canal de drenaje en tierra para estos ríos (Canal Sur) que fue ampliado y revestido en concreto hacia 1987; el canal en sus inicios coincidía con la cuenca baja del río Cañaveralejo, interceptaba el río Meléndez y posteriormente el río Lilí atravezaba la zona de Navarro en el sur-oriente de la ciudad y finalmente descargaba sus aguas al río Cauca, $11.8 \mathrm{Km}$ aguas arriba de las bocatomas de las plantas de tratamiento abastecidas por esta fuente.

Durante la década de los 60 's, la ciudad continuó expandiéndose principalmente en la zona plana, convirtiéndose el río Cauca en la principal fuente de abastecimiento, lo que evidenció la necesidad de ampliar el sistema de distribución 
para dar cobertura a nuevas zonas de expansión y equilibrar hidráulicamente el sistema, por lo que Empresas Municipales en 1960 contrató la firma americana Pitometer para los respectivos estudios, construyéndose importantes tanques de almacenamiento como el segundo tanque de La Normal, el primero de Siloé y el de La Campiña, además de la extensión y reposición de redes y construcción de estaciones de bombeo (Vásquez et al, 1995).

El establecimiento público Empresas Municipales de Cali - EMCALI como organismo autónomo, se creó en diciembre de 1961 (Acuerdo $\mathrm{N}^{\circ}$ 50) (Mercado, 2006) permitiéndole contraer obligaciones económicas que facilitaron la financiación de obras (Vásquez et al, 1995); en ese mismo año el Concejo Municipal le transfirió el manejo del alcantarillado de la ciudad por disponer de mayor capacidad técnica que el Municipio, quedando éstas a cargo del acueducto municipal, la energía eléctrica, los teléfonos, las plazas de mercado y de ferias, el matadero municipal y el alcantarillado (Vasquez, 2008).

En1963seconstruyóeltanquedealmacenamiento en Menga para el abastecimiento del norte de la ciudad con su respectiva estación de bombeo. Hacia 1969, EMCALI contaba con 17 tanques $\left(102,780 \mathrm{~m}^{3}\right)$ de los cuales nueve eran usados para equilibrar la demanda horaria en la zona alta y ocho en la zona baja; para abastecer las zonas altas y más alejadas de la ciudad existían cinco estaciones de bombeo (Vásquez et al, 1995, Vásquez 2008).

En 1964, EMCALI contrató la firma Hazen and Sawyer para la ampliación a $2.5 \mathrm{~m}^{3} / \mathrm{s}$ de la Planta Río Cauca (Vásquez, 2008), construcción que inició en 1967 y culminó en 1970; el estudio recomendó la construcción de una tercera planta de tratamiento en tres etapas de $3.3 \mathrm{~m}^{3} / \mathrm{s}$ cada una (Vasquez et al, 1995) y la ampliación de la capacidad de almacenamiento de la red baja con 4 tanques en Nápoles que deberían ser construidos por etapas a medida que la demanda lo exigiera y de los cuales solo hasta el año 2000 se construyeron 2 con capacidad de $15,000 \mathrm{~m}^{3} \mathrm{c} / \mathrm{u}$.
En diciembre de 1976 se inauguró la Planta de Tratamiento Puerto Mallarino $\left(3.3 \mathrm{~m}^{3} / \mathrm{s}\right)$, diseño a cargo del Ing. Hernando González Hurtado y la firma Hazen and Sawyer bajo la dirección del Ing. Herbert Hudson, que entró en funcionamiento en 1978 (Patiño, 2008; Camacho, 2006). El advenimiento de Puerto Mallarino hizo posible la extensión de la ciudad hacia lugares extremos como Ciudad Jardín, Limonar y toda la zona sur circundante, al igual que el extremo norte, además de satisfacer la demanda industrial y urbana de Yumbo (Camacho, 2006). Entre 1972-1978, se realizaron obras adicionales como la ampliación de la Planta San Antonio $\left(1.8 \mathrm{~m}^{3} / \mathrm{s}\right)$ implementando sedimentación laminar en placas, la instalación de tuberías de transmisión al sur y norte a partir de la planta Puerto Mallarino, la bocatoma nueva en el río Cauca con capacidad de bombeo de $12 \mathrm{~m}^{3} / \mathrm{s}$ (Vásquez, et al 1995), la ampliación de las estaciones de bombeo de Terrón Colorado, Siloé y Bellavista, la construcción del tanque de la Campiña $\mathrm{N}^{\circ} 2$ y la estación de bombeo de Nápoles tipo Booster (Vásquez et al, 1995).

En la década de los $80^{\prime}$ s, la ciudad empezó nuevamente a tener problemas de abastecimiento en sitios críticos como el distrito de Aguablanca y sectores de ladera en la zona sur y noroccidental y el índice de agua no contabilizada llegó a niveles muy elevados que hicieron que la demanda fuera superior a la oferta del servicio de acueducto, por lo que entre 1979 y 1985 se construyeron obras para solucionar estas deficiencias y ampliar la cobertura de las redes de distribución: abastecimiento de agua a Yumbo, almacenamiento para la zona suroccidental de Cali (Ciudad Jardín y Universidad del Valle - Sede Meléndez), interconexión de las redes alta y baja a través de la estación de bombeo la Normal para garantizar el suministro en verano, redes en barrios populares, continuación de instalaciones domiciliarias y reforestación y manejo de las subcuencas afluentes a Cali (EMCALI, (1981) citado por Camacho, 2006).

A partir de 1981, el río Cauca empezó a ser controlado por la CVC de forma periódica en 19 estaciones de monitoreo y como parte del proyecto de regulación del río, se dio inicio 
a la construcción del embalse Salvajina en el municipio de Suárez al norte del Departamento del Cauca, el cual entró en operación en Septiembre de 1985 (Sandoval \& Ramírez, 2007) con tres objetivos básicos i) controlar inundaciones, ofreciendo protección contra las crecientes en un periodo de retorno de 30 años, ii) aportar un caudal apropiado para aliviar la contaminación del río, asegurando un caudal mínimo de $130 \mathrm{~m}^{3} / \mathrm{s}$ en la estación de Juanchito y iii) generar electricidad con una capacidad instalada de $270 \mathrm{MW}$ (CVC, 2006).

Con el tiempo, se observó que las características hidráulicas del río Cauca cambiaron con la operación del embalse Salvajina; la curva de ascenso de la turbiedad antes de su construcción era lenta e iniciaba y culminaba después de 3 o 4 horas (característica típica de río de planicie) $\mathrm{y}$ con el funcionamiento del embalse, el ascenso de la turbiedad era rápido y culminaba 30 minutos después (característica de un río de montaña) (EMCALI, 1987), comportamiento que mostró la necesidad de construir un desarenador adicional en Puerto Mallarino para optimizar el tratamiento. Entre 1986 y 1990, la mayor parte de las obras se destinó a instalación de redes para el Distrito de Aguablanca y se incluyó un programa de abastecimiento de agua con camiones cisterna a las zonas más deprimidas de la ciudad (Siloé, Lleras, Brisas de Mayo, Pueblo Joven, El Vergel, Petecuy I, II y III) (Camacho, 2006).

En 1988 se contrató con el Consorcio GOARH (Gandini y Orozco, Angel y Rodríguez e Hidrotec con la asesoría de Camp Dresser y Mackee de USA) el Estudio de Mejoras y Ampliaciones del Acueducto de Cali que se basó en el análisis de oferta y demanda del servicio de acueducto para la elaboración de diseños definitivos a 2005 y esquemas de desarrollo a 2015. En el marco de este estudio se realizaron importantes diseños (modificación de filtros y cámaras de distribución de caudales y coagulación de Puerto Mallarino, sistema de acueducto de La Reforma, Tubería Transmisión Oriental - TTO, entre otros) y se formularon acciones para mitigar la vulnerabilidad por sismos en plantas y estaciones de bombeo, nuevos programas de monitoreo de la calidad del agua y optimización del manejo de materias primas (cambio del uso de sulfato de aluminio granulado a líquido).

El estudio determinó que para 2015 se necesitarían $13.8 \mathrm{~m}^{3} / \mathrm{s}$ para satisfacer la demanda de agua y complementar la capacidad total del sistema de acueducto en $2.5 \mathrm{~m}^{3} / \mathrm{s}$ evaluando cuatro opciones i) continuidad de uso del río Cauca en su captación actual o desde Hormiguero, ii) fuentes del Pacífico (ríos Digua, Yurumangui, San Juan, Anchicaya, etc) ubicadas tras la cordillera Occidental, iii) fuentes del Sur (tributarios río Cauca: ríos Claro, Timba, Jamundí, etc.) y iv) agua subterránea (14 pozos profundos perforados cada $\mathrm{Km}$ en la margen izquierda del río Cauca entre Hormiguero y el Distrito de Aguablanca).

La Planta de Tratamiento La Reforma, abastecida por el río Meléndez $\left(1.0 \mathrm{~m}^{3} / \mathrm{s}\right)$, entró en operación en diciembre de 1993 para abastecer la zona de laderas de la ciudad (Comunas 18 y 20) con dos salidas, Siloé y Nápoles (Patiño, 2008). Debido a la insuficiencia del río Meléndez en época de estiaje, se hizo evidente la necesidad del trasvase de agua de red baja a red Reforma, ampliándose el sistema de bombeo de Siloé; sin embargo, por deficiencias hidráulicas del sistema de alimentación de los tanques de compensación Siloe 1 y 2, éstos no fueron suficientes para cubrir la totalidad del déficit que se presentaba en esa época.

El acelerado desarrollo del Distrito de Aguablanca a principios de la década de los 90, sumado al retraso en la construcción de la segunda etapa de la planta Puerto Mallarino por razones legales, motivó la construcción de 4 pozos profundos (Los Naranjos, Las Orquídeas, Guaduales y Decepaz) con capacidad total de $400 \mathrm{~L} / \mathrm{s}$ con posterior cloración como opción de abastecimiento en esta zona. En el periodo del presidente C. Gaviria (1990 - 1994), la crisis energética nacional provocó la suspensión diaria del servicio de abastecimiento por lo que la población empezó a almacenar el agua, dando origen a la oxidación de hierro y manganeso comúnmente presentes en el agua subterránea, lo que causó la coloración amarillenta del 
agua, condición que causó notorio desagrado y rechazo entre los consumidores, desvirtuando la calidad del agua y obligando a buscar otra solución de abastecimiento diferente a los pozos (PDA, 2008).

El 16 de Agosto de 1996 se presentó una falla en las válvulas de los equipos contra Golpe de Ariete en la Planta Río Cauca, lo que ocasionó la inundación de la estación de bombeo de agua tratada, sumergiendo todos los equipos y provocando la parada de la planta durante cuatro días. La Planta Puerto Mallarino, a pesar de tener capacidad para suplir la demanda de red baja, no pudo hacerlo debido a que las salidas (sur y norte) de las dos plantas no se encontraban interconectadas, obra que se realizó a principios de 1997 para aumentar la flexibilidad del sistema.

Después de superar problemas legales que impedían la construcción de la segunda etapa de Puerto Mallarino, entró en servicio en 1997 una tercera línea de impulsión de agua tratada (tubería de transmisión oriental - TTO) y se dobló la capacidad de las estructuras con excepción de la batería de desarenación, considerada innecesaria desde la década de los 80 's por las características del río Cauca de tipo planicie; sin embargo, desde 1995 se contó con el diseño de esta estructura cuya construcción inició en el 2006 y entró en operación a finales del 2008. Con la puesta en funcionamiento de esta segunda etapa, los pozos salieron definitivamente de operación, quedando destinados solo para atender emergencias.

El creciente deterioro de la calidad del agua del río Cauca, notoria desde principios de la década del 90, ocasionó que los procesos de tratamiento convencional tuvieran que ser reforzados con tratamientos específicos; el derrumbe del botadero de Navarro sobre el Canal Sur en Septiembre de 2001, que descargó una elevada cantidad de lixiviados aguas arriba de la captación, hizo evidente el riesgo sanitario, obligando desde ese momento al uso continuo de la adsorción con Carbón Activado en Polvo (CAP) como barrera de tratamiento en las Plantas Puerto Mallarino y Río Cauca.
En Marzo de 2006, EMCALI EICE ESP asumió la operación de la planta La Rivera, que abastece sectores de Pance y completando con ésta las cinco plantas de tratamiento que actualmente están a su cargo. Esta planta contaba con una capacidad de $14 \mathrm{~L} / \mathrm{s}$ y tecnología FIME pero debido a la rápida expansión del sector, fue necesaria su ampliación a $34 \mathrm{~L} / \mathrm{s}$ y al cambio de tecnología, siendo la filtración rápida y floculación-clarificación en medio granular la opción seleccionada.

El incremento de la contaminación orgánica y de la turbiedad en el río Cauca, ha ocasionado cambios drásticos tanto de caudal como de calidad dependiendo de la época del año, ratificando un comportamiento característico de río de montaña. El mayor número de paradas de las plantas abastecidas con esta fuente por eventos extremos de carga contaminante (Oxígeno Disuelto $<2.5 \mathrm{mgO}_{2} / \mathrm{L}$ ) y picos de turbiedad (>2500 UNT) han afectado la calidad y continuidad del servicio (Escobar, 2010; Montoya et al, 2011), causando inconformidad en los usuarios y deterioro de la imagen pública de la empresa, presentándose la mayor crisis en el año 2007 que hizo que EMCALI EICE ESP decidiera iniciar la construcción de un reservorio de agua clarificada que entró en operación desde Octubre del 2009 y se ubicó en la zona de ampliación de la tercera etapa de la planta Puerto Mallarino, con una capacidad de 80,000 m3 para ser operado en eventos de elevada carga contaminante o combinado con agua cruda en eventos de elevada turbiedad, sirviendo como medida de control del riesgo sanitario (Escobar et al, 2010).

El SAAP de la ciudad de Cali cuenta con 5 plantas de tratamiento abastecidas por 4 fuentes superficiales (capacidad total instalada 11.93 $\mathrm{m}^{3} / \mathrm{s}$ de la cual se utiliza cerca del $61 \%$ ). El sistema de distribución de agua potable está dividido en 4 redes con 2,820 $\mathrm{Km}$ de tuberías, 41 tanques de almacenamiento y 16 estaciones de bombeo para la impulsión del agua hacia las partes más elevadas y lejanas de la ciudad; la Tabla 2 muestra la descripción general del SAAP en la actualidad. 
Tabla 2. Descripción general del SAAP de la ciudad de Cali

\begin{tabular}{|c|c|c|c|c|c|c|c|}
\hline $\begin{array}{c}\text { Fuente de } \\
\text { abastecimiento }\end{array}$ & \multicolumn{5}{|c|}{ Planta de tratamiento } & $\begin{array}{l}\text { Sistema de } \\
\text { distribución }\end{array}$ & $\begin{array}{c}\text { Población } \\
\text { abastecida \% }\end{array}$ \\
\hline RIO CALI & Río Cali & Ciclo completo & 1930 & 1.8 & 1.29 & Red Alta & 17.1 \\
\hline RIO CAUCA & Río Cauca & Ciclo completo $^{1}$ & 1958 & 2.5 & 1.53 & Red Baja & 21.0 \\
\hline RIO CAUCA & Puerto Mallarino & Ciclo completo $^{1}$ & 1978 & 6.6 & 4.03 & Red Baja & 56.0 \\
\hline \multirow[t]{2}{*}{ RIO PANCE } & Rivera & Ciclo completo & 19933 & 0.034 & 0.014 & Red Pance & 0.2 \\
\hline & Total & & & & 11.93 & 7.23 & 100 \\
\hline
\end{tabular}

${ }^{1}$ Más tratamientos específicos (Adsorción con Carbón Activado en Polvo - CAP); ${ }^{2}$ La planta también puede operar en la modalidad de filtración directa y en línea; ${ }^{3}$ Operada por EMCALI EICE ESP desde 2006; ${ }^{4}$ Datos año 2010

\section{Perspectivas frente al aseguramiento de la calidad del agua potable}

Una de las problemáticas que afecta en los últimos años la continuidad del suministro de agua potable de la ciudad, ha sido el progresivo deterioro de la cuenca de abastecimiento del río Cauca que continúa causando eventos extremos de contaminación $(\mathrm{OD}<2.5 \mathrm{mg} / \mathrm{L})$ y elevada turbiedad $(>2,500$ UNT) que como una medida de prevención del potencial riesgo sanitario, obligan al cierre de la captación de agua.

Por estas razones, además de la construcción del reservorio de agua clarificada en la Planta Puerto Mallarino y la existencia de una estación de alerta temprana aguas arriba de la captación (Estación Milán) como medidas de control para garantizar la continuidad del servicio (Escobar et al, 2010), se han explorado de forma preliminar alternativas como la reubicación de la captación en una zona con mejor calidad de agua, construcción de un embalse a la altura del río Pichindé y uso de fuentes alternas de abastecimiento como el río Timba y el agua subterránea (considerada principalmente para condiciones de emergencia y contingencia) (El tiempo, 2011) mientras se logran a largo plazo los resultados de la iniciativa del gobierno por recuperar el río Cauca establecidas en el CONPES 3624 (MAVDT et al, 2009).

EMCALI EICE ESP, entidad responsable de la prestación del servicio de acueducto y alcantarillado de la ciudad, a lo largo de su historia ha respondido al constante requerimiento de agua, cumpliendo con el objetivo social de garantizar la salud de la población mediante el suministro de agua en calidad y cantidad adecuadas e incrementando su cobertura a medida que la ciudad lo ha demandado. Este compromiso también se ratifica con el análisis estadístico de la información histórica de calidad del agua monitoreada en el sistema de distribución, la cual evidencia el cumplimiento de la reglamentación nacional - Resolución 2115/07 (MAVDT y MPS, 2007) en más del 99\% de los datos, presentando un nivel sin riesgo a la salud según los valores del Índice de Riesgo de Calidad del Agua (IRCA) (Pérez et al, 2012).

La empresa de servicios públicos, permaneciendo a la vanguardia de las tendencias mundiales del sector de agua potable y saneamiento, viene desarrollando una herramienta integral de evaluación y gestión del riesgo denominada Plan de Seguridad del Agua (PSA) que garantiza la salud de la población mediante el aseguramiento de la calidad del agua desde la cuenca de abastecimiento hasta el usuario final (WHO, 2011). Para ello, ha realizado la sistematización de los peligros y eventos peligrosos en cada uno de los componentes del SAAP (cuenca de abastecimiento, procesos de tratamiento, sistema de distribución y usuario final), la evaluación del riesgo apoyada en herramientas como matrices de riesgo semicuantitativas y Sistemas de Información Geográfica que han permitido la construcción de mapas de peligros y riesgos (Torres et al, 2010; Torres et al, 2011; Pérez et al 2012) y la redefinición de la red de monitoreo de calidad de agua potable en el sistema de distribución (Montoya et al, 2009) dando 
cumplimiento a las reglamentaciones vigentes, Resolución 4716/2010 (MAVDT, 2010) y 0811/2008 (MAVD, 2008c) respectivamente.

El desarrollo del PSA ha sido un proceso dinámico, que también ha permitido la formulación de planes de mejoramiento $\mathrm{y}$ optimización de medidas de control y programas de soporte orientados a la capacitación, fortalecimiento de las capacidades analíticas y la investigación de nuevas medidas de control del riesgo y optimización de otras, apoyados principalmente en el convenio de cooperación interinstitucional entre el sector académico y la empresa (Torres et al, 2011). Con la implementación del Sistema de Gestión de la Calidad (SGC) y certificación bajo la norma ISO 9001:2000, también se han logrado avances significativos en el aseguramiento de la calidad del agua potable; adicionalmente, conscientes de la importancia del manejo de la información en el control de la calidad del agua, se ha adelantado en la sistematización e integración de la información del sistema mediante la base de datos SIDAP (Sistema de Información de Producción y Distribución de Agua Potable).

De forma complementaria, la empresa viene adelantando proyectos abordando la problemática del agua bajo el concepto de la Gestión Integral del Recurso Hídrico - GIRH, partiendo de la cuenca hidrográfica como la principal unidad de ordenamiento del territorio, trabajando en la fuente de abastecimiento, en los procesos de potabilización, en el sistema de distribución de agua potable, en el tratamiento de aguas residuales domésticas y el manejo integral de los biosólidos como se muestra en la Figura 2.

La implementación de enfoques como los PSA y la GIRH, ayudarán a mitigar en parte el progresivo deterioro de las fuentes de agua que de continuar, obligará al uso de procesos de tratamiento más sofisticados y costosos y a largo plazo hasta tener que prescindir del uso de esas fuentes, lo que podría conllevar a una crisis de desabastecimiento. Estos enfoques son aplicables a todo SAAP, independiente de su tamaño o complejidad y aunque los PSA deben ser formulados de manera específica, la existencia de experiencias piloto facilita su desarrollo y replicación en otros SAAP (Davison y Deere, 2007), siendo indispensable contar con el compromiso y apoyo de los tomadores de decisiones por las implicaciones técnicas y financieras que pueden presentarse (DWI, 2005;

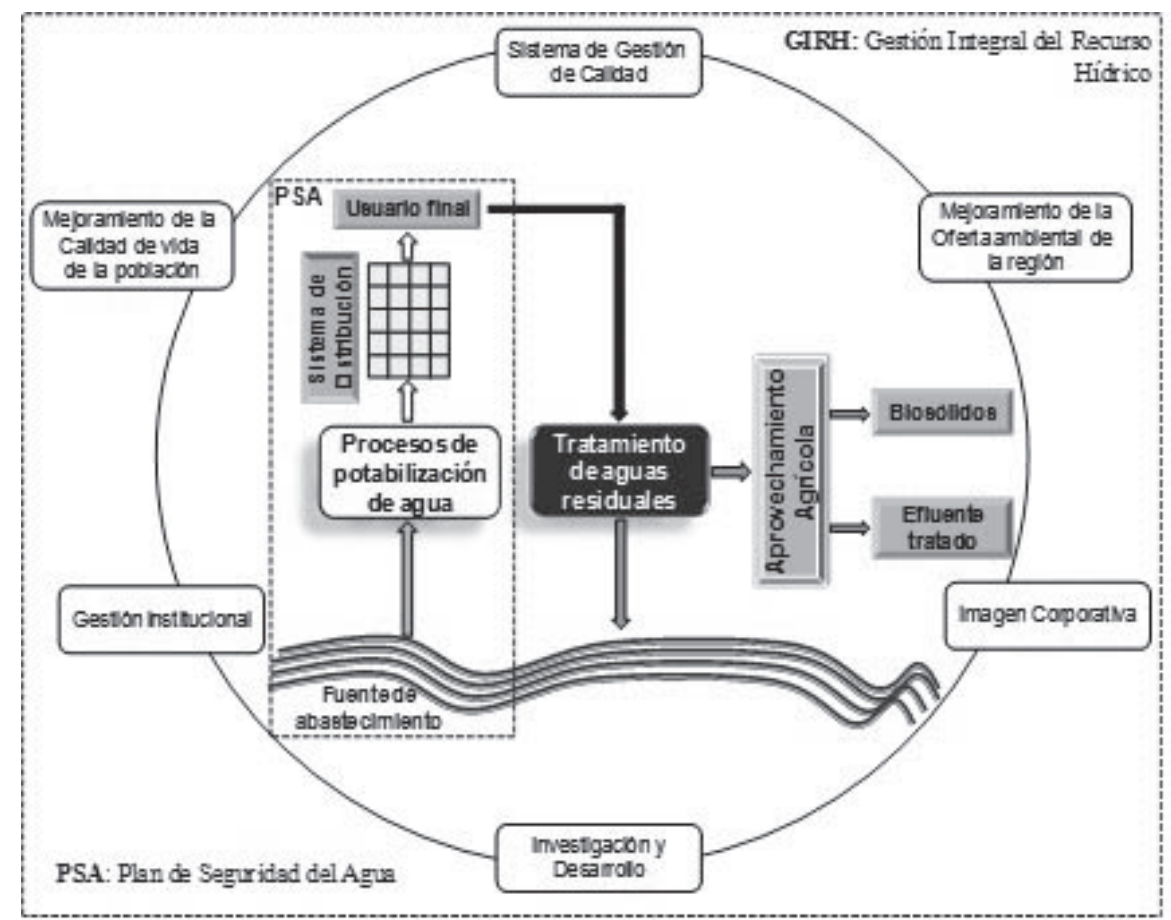

Figura 2. Problemática del agua abordada desde la perspectiva de la Gestión Integral del Recurso Hídrico 
SPC, 2011) y la participación de todos los actores del SAAP (usuarios del suelo en la cuenca de abastecimiento, entidades ambientales y municipales, empresas prestadoras del servicio de agua, entidades de salud pública, usuarios del servicio de agua) que garantice un trabajo interinstitucional coordinado.

Considerando que el acceso al agua potable es un bien esencial que favorece el progreso de las comunidades mejorando su calidad de vida, es fundamental que la misión de las entidades responsables de la prestación del servicio de agua gire en torno al bienestar de la población con el suministro de agua segura, siendo la experiencia del SAAP de la ciudad de Cali un ejemplo con gran potencial de adaptación y aplicación a otros sistemas de abastecimiento del país que estén encaminados a mejorar la gestión del riesgo como estrategia de aseguramiento de la calidad del agua potable.

\section{Conclusiones}

El análisis histórico del Sistema de Abastecimiento de la ciudad de Cali, evidencia que el progresivo deterioro de las fuentes de agua, causado principalmente por actividades antrópicas, ha obligado a la incorporación de barreras adicionales de tratamiento y la implementación de tecnologías robustas para garantizar la calidad del agua potable, situación que es generalizada en el país y que requiere fomentar y mejorar el trabajo interinstitucional de entidades ambientales, de salud y prestadoras del servicio de agua como estrategia para el mejoramiento de la oferta hídrica y el aseguramiento de la calidad del agua suministrada al usuario final.

EMCALI EICE ESP, como empresa prestadora del servicio de agua para la ciudad de Cali, ha respondido satisfactoriamente a los cambios que ésta ha demandado, implementando permanentemente medidas preventivas $\mathrm{y}$ correctivas para garantizar siempre agua segura a los usuarios, anteponiendo su compromiso ético y de responsabilidad social y contribuyendo al bienestar y desarrollo de la comunidad y protección del ambiente. Los registros históricos de calidad de agua potable evidencian el cumplimiento de la reglamentación nacional, con valores del Índice de Riesgo de Calidad del Agua que clasifican el agua como "sin riesgo a la salud".

Es importante que los sistemas de abastecimiento de agua desarrollen e implementen estrategias preventivas para gestionar los riesgos; la metodología de los Planes de Seguridad del Agua (PSA) permite una mejor comprensión del sistema mediante la identificación de los eventos peligrosos y medidas de control, evaluación del riesgo y formulación de programas de mejoramiento, modernización o implementación de nuevas medidas de control como parte de la gestión del riesgo. La aplicabilidad de esta metodología se extiende a todos los SAAP, siendo posible el uso de herramientas sencillas (Ej. visitas de inspección sanitaria, matrices semicuantitativas) $\mathrm{y} / \mathrm{o}$ de mayor complejidad (Ej. Mapas de riesgo usando Sistemas de Información Geográfica) para las etapas de identificación de peligros y evaluación del riesgo; la selección del nivel de complejidad dependerá de la disponibilidad de información y la capacidad de recursos técnicos $\mathrm{y}$ financieros disponibles. Adicionalmente, el monitoreo y seguimiento sistemático de las medidas de control del riesgo garantizará el adecuado funcionamiento del PSA facilitando su ajuste y actualización periódica.

\section{Agradecimientos}

Los autores agradecen a COLCIENCIAS, EMCALI EICE ESP y la Universidad del Valle por el apoyo financiero. Se hace un reconocimiento especial al Ing. Alejandro Pérez Duarte, ex jefe Depto. Producción Agua Potable de EMCALI EICE ESP por suministrar valiosa información para elaborar este artículo.

\section{Referencias bibliográficas}

Aladino. (1992). Acueducto y Alcantarillado Cali 1900-1970. Investigación presentada al concurso historia servicios públicos de Cali. Estudios territoriales coordinación Planeación y Desarrollo EMCALI. 
Camacho, M.G. (2006). EMCALI en la historia de Cali. ISBN 958-33-9245-6. Cali: Empresas municipales de Cali EMCALI EICE ESP.

Centro virtual Isaacs. (2004). La navegación a vapor por el río Cauca. Portal Cultural del Pacífico. http://dintev.univalle.edu.co/cvisaacs

CVC (Corporación Autónoma Regional del Valle del Cauca). (2006). Multimedia Sistema de Regulación del Río Cauca. http://www. cvc.gov.co/vsm38cvc/data/RecursoHidrico/ multimedias/Salvajina/Menu.swf

CVC (Corporación Autónoma Regional del Valle del Cauca). (2011). Así nació la CVC. Nuestra historia. http://www.cvc.gov.co/ vsm38cve/

Directorio Telefónico. (2005-2006). El Agua, 75 años de la planta de potabilización río Cali de San Antonio. Santiago de Cali.

DNP (Departamento Nacional de Planeación). (2009). Programa para el saneamiento, manejo y recuperación ambiental de la cuenca alta del río Cauca. Documento CONPES 3624. Noviembre 20, Bogotá D.C, República de Colombia.

El Tiempo. (2011). A 300 metros buscan el agua para contingencias. Septiembre 5. Sección Cali. p.13.

EMCALI (Empresas Municipales de Cali). (s.f). Origen y Desarrollo - Reseña Histórica. Artículo publicado por la Gerencia de Acueducto y Alcantarillado en revista de divulgación (Década del 90's).

EMCALI (Empresas Municipales de Cali). (1981). Informe de gerencia General, Cali. Citado por Camacho, 2006.

EMCALI (1987). Salvajina y comportamiento de la calidad del agua del río Cauca. Correspondencia interna Sección Operativa Planta Puerto Mallarino - Departamento Producción de agua potable. Mayo 29.

Escobar, J.C. (2010). Riesgo en calidad de agua potable asociado a eventos extremos de turbiedad y carga contaminante. En: Memorias
Seminario de Gestión Integral del Riesgo en Sistemas de Agua Potable y Saneamiento, Bogotá D.C, Colombia, Marzo 4-5, Colombia: ACODAL - ANDESCO.

Escobar, J.C., Torres, P., Cruz, C., \& Pérez, A. (2010). Impacto en el aseguramiento de la calidad del agua potable por eventos extremos de caída de oxígeno disuelto en la fuente de abastecimiento. Asociación Interamericana de Ingeniería Sanitaria y Ambiental. En: Memorias XXXII Congreso Interamericano de Ingeniería Sanitaria y Ambiental AIDIS, Punta Cana, , República Dominicana.

Godfrey, S., \& Howard, G. (2005). Water Safety Plans: Book 1. Planning Water Safety Management for Urban Piped Water Supplies in Developing Countries. WEDC, Loughborough University, Inglaterra. http://wedc.lboro.ac.uk/ publications/index.htm

Magazine Despertar Vallecaucano. (1976). El acueducto de Cali un esfuerzo de los grandes caleños. Número 26. Marzo -Abril.

MAVDT (Ministerio Ambiente, Vivienda y Desarrollo Territorial) \& MPS (Ministerio de Protección Social). (2007). Resolución 2115 de 2007, por medio de la cual se señalan características, instrumentos básicos y frecuencias del sistema de control y vigilancia para la calidad del agua para consumo humano. República de Colombia.

MAVDT (Ministerio de Ambiente, Vivienda y Desarrollo Territorial). (2008a). Foro Vida. Noticias 190608, Bogotá, Junio.

MAVDT (Ministerio de Ambiente, Vivienda y Desarrollo Territorial). (2008b). Agua Transparente. Noticias 230508, Bogotá, Mayo.

MAVDT (Ministerio de la Ambiente, Vivienda y Desarrollo Territorial). (2008c). Resolución No. 0811 de 2008, por medio del cual se establece el control y vigilancia de la calidad del agua potable. República de Colombia.

MAVDT (Ministerio de Ambiente, Vivienda y Desarrollo Territorial). (2010). Resolución 4710 de 2010, por medio de la cual se reglamenta el 
parágrafo del artículo 15 del Decreto 1575 de 2007. República de Colombia.

Mercado, C. (2006). Recopilación e investigación sobre la historia del acueducto de Cali. Centro de Estudios Históricos y Sociales, Santiago de Cali.

Montoya, C., Loaiza, D., Cruz, C., Torres, P., Escobar, J.C., \& Delgado, L.G. (2009). Propuesta metodológica para localización estaciones monitoreo calidad de agua en redes de distribución utilizando sistemas de información geográfica. Revista Facultad de Ingeniería 49,129-140.

Montoya, C., Loaiza, D., Torres, P., Cruz, C. \& Escobar, J.C. (2011). Efecto del incremento en la turbiedad del agua cruda sobre la eficiencia de procesos convencionales de potabilización. Revista EIA 16, 137-148.

OMS (Organización Mundial de la Salud). (2004). Guidelines for drinking water quality. Third edition. Vol 1. Geneva.

OMS (Organización Mundial de la Salud). (2007). Lucha contra las enfermedades transmitidas por el agua en los hogares. Red Internacional para la promoción del tratamiento y almacenamiento seguro del agua doméstica. http://www.bvsde.paho.org/bvsacd/cd68/ combating/combating.html

OMS (Organización Mundial de la Salud). (2011). Guidelines for Drinking-water Quality. Fourth edition.

Patiño, S. (2008). Agua en la vida de Cali. Cali, Spatiño.

PDA (Plan Departamental del Agua). (2007). Diagnóstico técnico de acueducto y alcantarillado en el área urbana. Valle del Cauca: Alcaldía Santiago de Cali.

Pérez, A. (2006). Historia del acueducto de Cali. Conferencia presentada a la sociedad civil en conmemoración de los 75 años de la planta de Río Cali. Diciembre. Cali, Colombia.
Pérez, A., Amézquita, C., \& Torres, P. (2012). Identificación y priorización de peligros en sistemas de distribución de agua potable usando sistemas de información geográfica. Revista Ingeniería y Universidad 16 (2), 449-469.

Rojas, R. (2006). Planes de seguridad del agua (PSA). OMS/OPS/SDE/CEPIS-SB, Hojas de Divulgación Técnica, HDT $-\mathrm{N}^{\circ}$ 100. http:// www.bvsde.ops-oms.org/bvsair/e/hdt/hdt100/ hdt100.pdf

Sandoval, M., \& Ramírez, C. (2007). El río Cauca en su valle alto. Un aporte al conocimiento de uno de los ríos más importantes de Colombia. Colombia: Universidad del Valle- CVC.

Torres, P., Pérez, A., Escobar, J.C., \& Cruz, C. (2010). Identificación de peligros en la fuente de abastecimiento en la ciudad de Cali enmarcada en la formulación de un plan de seguridad del agua. En: XXXII Congreso Interamericano de Ingeniería Sanitaria y Ambiental AIDIS. Punta Cana, Noviembre, República Dominicana.

Torres, P., Pérez, A., Cruz, C., Delgado, L.G., \& Escobar, J.C. (2011). Planes de seguridad del agua como estrategia de gestión del riesgo en los sistemas de abastecimiento de agua potable. Caso: Cali - Colombia. En: $54^{\circ}$ Congreso de ACODAL. Santa Marta, Agosto 31- Septiembre 1-2, Colombia: ACODAL.

Vásquez, E. (2001). Historia de Cali en el Siglo 20. Sociedad, economía, cultura y espacio. Cali, Colombia

Vásquez, E. (2008). Acueducto siglo XX: tránsito a la modernización. En: S. Patiño (editor), Agua en la vida de Cali. Sylvia Patiño.

Vásquez, E., Corchuelo, A., Escobar, J. \& Bayona, A. (1995). Descripción histórica del desarrollo del acueducto de Cali. EMCALI - Gerencia de Planeación y Desarrollo Centro de investigaciones y documentación socioeconómica Facultad de ciencias sociales y económicas Universidad del Valle. 